\title{
Screening for Cryptococcal Antigenemia and Burden of Cryptococcosis at the Time of HIV Diagnosis: A Retrospective Multicenter Study
}

\author{
Sung-Hsi Huang $\cdot$ Chun-Yuan Lee $\cdot$ Chin-Shiang Tsai $\cdot$ Mao-Song Tsai $\cdot$ Chun-Eng Liu $\cdot$ Wei-Ting Hsu • \\ Hong-An Chen · Wang-Da Liu $\cdot$ Chia-Jui Yang $\cdot$ Hsin-Yun Sun $\cdot$ Wen-Chien Ko $\cdot$ Po-Liang Lu • \\ Yuan-Ti Lee $\cdot$ Chien-Ching Hung (10
}

Received: February 28, 2021 / Accepted: April 22, 2021 / Published online: May 29, 2021

(c) The Author(s) 2021

\section{ABSTRACT}

Introduction: Screening for cryptococcal antigen (CrAg) is recommended for people living with HIV (PLWH) who present with low CD4 lymphocyte counts. Real-world experience is

Yuan-Ti Lee, Chien-Ching Hung have contributed equally to the work and should be considered joint senior authors.

The members of the Taiwan HIV Study Group are mentioned in the "Acknowledgements" section.

Supplementary Information The online version contains supplementary material available at https://doi. org/10.1007/s40121-021-00451-5.

\section{S.-H. Huang}

Department of Internal Medicine, National Taiwan University Hospital Hsin-Chu Branch, Hsin-Chu, Taiwan

S.-H. Huang · C.-C. Hung ( $\bowtie)$

Department of Tropical Medicine and Parasitology, National Taiwan University College of Medicine, Taipei, Taiwan e-mail: hcc0401@ntu.edu.tw

S.-H. Huang e-mail: b87401012@ntu.edu.tw

C.-Y. Lee · P.-L. Lu

Department of Internal Medicine, Kaohsiung Medical University Hospital and College of Medicine, Kaohsiung Medical University, Kaohsiung, Taiwan important to identify gaps between the guidelines and clinical practice. We investigated the trends of CrAg testing and prevalence of cryptococcal antigenemia among PLWH at the time of HIV diagnosis and the related mortality in Taiwan from 2009 to 2018.

Methods: Medical records of newly diagnosed PLWH seeking care at six medical centers around Taiwan between 2009 and 2018 were reviewed. The annual trends of PLWH who had CrAg testing and cryptococcal antigenemia were examined by Cochran-Armitage test. Among PLWH with CD $4<200$ cells/ $\mu$ l, timing of CrAg testing was analyzed for association with 12-month all-cause mortality in Kaplan-

\author{
C.-Y. Lee \\ e-mail: leecy8801131@gmail.com \\ P.-L. Lu \\ e-mail: idpaul@gmail.com \\ C.-S. Tsai · W.-C. Ko \\ Department of Internal Medicine, National Cheng \\ Kung University Hospital, College of Medicine, \\ National Cheng Kung University, Tainan, Taiwan \\ C.-S. Tsai \\ e-mail: jasonmammal@gmail.com \\ W.-C. Ko \\ e-mail: winston@mail.ncku.edu.tw
}


Meier plots and in a Cox proportional hazards model after adjustments.

Results: Among 5372 included PLWH, 1150 (21.4\%) presented with baseline CD4 $<100$ cells $/ \mu \mathrm{l}$, and this proportion had decreased during the study period [from $108(29.3 \%)$ in 2009 to $93(22.3 \%)$ in $2018(P=0.039)]$. The overall prevalence of cryptococcal antigenemia was $7.8 \%$ among PLWH with CD4 $<100$ cells/ $\mu \mathrm{l}$, which remained stable during the 10-year study period $(P=0.356)$ and was $2.6 \%$ among PLWH with CD4 100-199 cells/ $\mu$ l. The uptake of CrAg testing had increased from $65.7 \%$ in 2009 to $78.0 \%$ in $2018(P=0.002)$ among PLWH with CD $4<100$ cells/ $\mu$ l. Late CrAg testing, defined by 14 days or later after HIV diagnosis, was associated with increased risk of 12-month mortality compared to early CrAg testing (adjusted hazard ratio 2.028, 95\% CI 1.109-3.708).

M.-S. Tsai · H.-A. Chen · C.-J. Yang

Department of Internal Medicine, Far Eastern

Memorial Hospital, New Taipei City, Taiwan

H.-A. Chen

e-mail: joseph2300571@gmail.com

M.-S. Tsai

School of Medicine, College of Medicine, Fu Jen

Catholic University, New Taipei City, Taiwan

e-mail: tmao.song@gmail.com

C.-E. Liu

Department of Internal Medicine, Changhua

Christian Hospital, Changhua, Taiwan

e-mail: chuneng@cch.org.tw

W.-T. Hsu

Department of Internal Medicine, National Taiwan

University Hospital Yun-Lin Branch, Douliu, Yun-

Lin County, Taiwan

e-mail: b95401065@ntu.edu.tw

W.-D. Liu · H.-Y. Sun · C.-C. Hung

Department of Internal Medicine, National Taiwan University Hospital, National Taiwan University College of Medicine, 7 Chung-Shan South Road,

Taipei, Taiwan

H.-Y. Sun

e-mail: hysun13@gmail.com
Conclusions: Burden of cryptococcosis remained high among PLWH with low CD4 lymphocyte counts in Taiwan. Uptake of CrAg screening among late HIV presenters was still suboptimal and delayed. Late CrAg testing was associated with a higher mortality.

Keywords: Opportunistic infections; Cryptococcal antigen; Cryptococcal meningitis; People living with HIV; Late presenter; Care cascade

W.-D. Liu

Department of Medicine, National Taiwan University Cancer Center, Taipei, Taiwan e-mail: b95401043@ntu.edu.tw

C.-J. Yang

School of Medicine, National Yang-Ming ChiaoTung University, Taipei, Taiwan

e-mail: yangcj1206@gmail.com

Y.-T. Lee

School of Medicine, Chung Shan Medical

University, Taichung, Taiwan

Y.-T. Lee

Department of Internal Medicine, Chung Shan Medical University Hospital, Taichung, Taiwan e-mail: leey521@gmail.com

C.-C. Hung

Department of Medical Research, China Medical University Hospital, Taichung, Taiwan

C.-C. Hung

China Medical University, Taichung, Taiwan 


\section{Key Summary Points}

\section{Why carry out this study?}

Screening for cryptococcal antigenemia at the time of HIV diagnosis is recommended for people living with HIV who present with low CD4 lymphocyte counts

Uptake of cryptococcal antigen screening and prevalence of cryptococcosis in

Taiwan in the era of universal antiretroviral therapy are unknown

\section{What was learned from the study?}

Prevalence of cryptococcal antigenemia at the time of HIV diagnosis was $7.8 \%$ and $2.6 \%$ among people living with HIV who presented with CD4 lymphocyte counts < 100 and 100-199 cells/ $\mu$ l, respectively

Uptake of cryptococcal antigen screening among HIV late presenters was improving, but still suboptimal and delayed, and this delay was associated with a higher mortality

Delayed cryptococcal antigen screening was associated with mortality and hospitalization among people living with HIV who were diagnosed in the outpatient settings and presented with initial CD4 lymphocyte counts $<200$ cells/ $\mu \mathrm{l}$

\section{DIGITAL FEATURES}

This article is published with digital features, including a summary slide, to facilitate understanding of the article. To view digital features for this article go to https://doi.org/10.6084/ m9.figshare.14459733. 
unknown. In this study, we aimed to investigate the uptake of CrAg testing among newly diagnosed PLWH in Taiwan between 2009 and 2018 , the prevalence of cryptococcal antigenemia, and their temporal changes. We also explored the impact of the timing of $\mathrm{CrAg}$ testing on all-cause mortality and hospital admission among those who presented with a CD4 lymphocyte count of $<200$ cells $/ \mu$ l.

\section{METHODS}

\section{Study Setting and Patients}

In this retrospective, multicenter study, consecutive PLWH who were newly diagnosed with HIV infection and presented for care to six medical centers around Taiwan for the first time between 2009 and 2018 were included. Those who had received their HIV diagnosis outside of these six participating hospitals and those who refused HIV care or were lost to follow-up shortly after a confirmed HIV diagnosis were excluded. Information on the demographics, co-infections, and results of laboratory tests at the time of HIV diagnosis was collected, as well as the first serum cryptococcal antigen titer within 6 months before and after the HIV diagnosis. Clinical outcomes, including retention in care, hospital admission, and mortality, were tracked up to 12 months after the HIV diagnosis.

The study was approved by the Research Ethics Committees and Institutional Review Boards of the participating hospitals (National Taiwan University Hospital, registration number 201003112R]; Far Eastern Memorial Hospital [105040-F]; Chung Shan Medical University Hospital [CS14034], Changhua Christian Hospital [160408]; National Cheng Kung University Hospital [B-BR-105-038]; Kaohsiung Medical University Hospital [KMUH-IRB20110040]), and the informed consent was waived. The study was carried out according to the principles expressed in the Declaration of Helsinki.

Routine baseline laboratory investigations for newly diagnosed PLWH in Taiwan included CD4 lymphocyte count, plasma HIV RNA load
(PVL), complete blood-cell count, liver and renal function tests, and serology for hepatitis $\mathrm{A}$ virus (HAV), hepatitis B virus (HBV), hepatitis C virus (HCV), and syphilis according to the national HIV treatment guidelines. The above laboratory investigations and prescription of ART are reimbursed by the Taiwan Centers for Disease Control. During the study period, ART initiation was recommended when CD4 was $<$ 200 cells $/ \mu$ l in 2009, when CD4 was $<350$ cells/ $\mu$, in 2010-2012, and when CD4 was $<500$ cells/ $\mu$ l in 2013-2015; after 2016, newly diagnosed PLWH were provided with ART regardless of $\mathrm{CD} 4$ count at diagnosis.

\section{Study Outcomes and Definitions}

In this study, the burden of cryptococcosis was represented by the prevalence of cryptococcal antigenemia, defined by positive CrAg irrespective of the titers, and that of CrAg titer $\geq 1: 8$, which indicated active cryptococcosis [16]; both prevalences were stratified according to CD4 lymphocyte count categories $(<100$, $100-199$, and $\geq 200$ cells $/ \mu \mathrm{l})$. Diagnosis of cryptococcal meningitis was confirmed by isolation of Cryptococcus species in the cerebrospinal fluid (CSF) or a CSF CrAg titer $\geq 1: 8$ [17].

To investigate the association between timing of CrAg testing and mortality in PLWH who presented with low CD4 counts, the included PLWH were categorized in the "late CrAg" group if CrAg was determined 14 days or later after HIV diagnosis [18], in the "early CrAg" group if CrAg was determined no later than 14 days after HIV diagnosis, and in "CrAg before HIV" group if $\mathrm{CrAg}$ was determined before confirmed HIV diagnosis. PLWH presented as "inpatients" at the time of HIV diagnosis if they were hospitalized when they received the HIV diagnosis; otherwise, they were classified as "outpatients."

\section{Laboratory Investigations}

Throughout the study period, Latex-Cryptococcus Antigen Test (IMMY, Oklahoma, USA) was employed to determine CrAg positivity and CrAg titers with dilution up to 1:1024 [19]. The 
procedure was carried out at each participating hospital according to the manufacturer's instructions. Determinations of PVL, CD4 lymphocyte count, and serological tests for syphilis and viral hepatitis (HAV, HBV, and HCV) were performed using certified commercial kits at the six participating hospitals.

\section{Statistical Analysis}

Proportions of newly diagnosed PLWH who underwent CrAg testing, as well as the prevalences of cryptococcal antigenemia and $\mathrm{CrAg}$ titer $\geq 1: 8$ among PLWH who tested for CrAg, were calculated annually and examined by Cochran-Armitage test for trend. Factors associated with CrAg testing among PLWH with CD $4<100$ cells/ $\mu$ l were examined in univariate analysis, with categorical variables analyzed by chi-square test or Fisher's exact test, and continuous variables by Mann-Whitney $\mathrm{U}$ tests. Variables with $P$-value $<0.1$ in the univariate analysis were then entered in multivariate logistic regression models with missing values treated by exclusion to identify independent association factors. The same procedure was carried out to identify factors associated with positive CrAg among those who tested for it.

Among PLWH with CrAg testing and baseline $\mathrm{CD} 4<200$ cells/ $\mu$ l, the 6 - and 12 -month all-cause mortality was shown on Kaplan-Meier plots, with stratification by timing of $\mathrm{CrAg}$ testing and inpatient/outpatient status at the time of HIV diagnosis. The association of timing of CrAg testing with 12-month all-cause mortality among PLWH who were outpatients at the time of HIV diagnosis was examined in a Cox proportional hazards model after adjusting for age, risk of HIV acquisition, baseline PVL and CD4 lymphocyte count, and presence of any opportunistic infection other than cryptococcosis. The association of timing of $\mathrm{CrAg}$ testing with 12-month all-cause hospital admission was also explored in the same manner. Statistical analyses were performed using the $\mathrm{R}$ statistics software (version 3.6.1), and $P<0.05$ was deemed statistically significant throughout the analyses.
Table 1 Baseline characteristics of 5372 newly diagnosed people living with HIV included in the study

\begin{tabular}{|c|c|}
\hline & $\begin{array}{l}\text { Total } \\
(N=5372)\end{array}$ \\
\hline Age, median (IQR), years & $29.4(24.5,36.0)$ \\
\hline$<20$ years, $n(\%)$ & $216(4.0)$ \\
\hline $20-29.9$ & $2620(48.8)$ \\
\hline $30-39.9$ & $1618(30.1)$ \\
\hline $40-49.9$ & $639(11.9)$ \\
\hline$\geq 50$ & $279(5.2)$ \\
\hline Male sex at birth, $n(\%)$ & $5227(97.3)$ \\
\hline \multicolumn{2}{|l|}{ Geographic location, $n(\%)$} \\
\hline Northern Taiwan & $2751(51.2)$ \\
\hline Central Taiwan & $798(14.9)$ \\
\hline Southern Taiwan & $1823(33.9)$ \\
\hline \multicolumn{2}{|l|}{ Risk group for HIV acquisition, $n$ (\%) } \\
\hline MSM & $4461(83.0)$ \\
\hline IDU & $235(4.4)$ \\
\hline \multicolumn{2}{|l|}{ Year of HIV diagnosis, $n(\%)$} \\
\hline 2009-2011 & $1316(24.5)$ \\
\hline $2012-2013$ & $1270(23.6)$ \\
\hline 2014-2015 & $1316(24.5)$ \\
\hline $2016-2018$ & $1470(27.4)$ \\
\hline $\begin{array}{l}\text { Being outpatients at the time of HIV } \\
\text { diagnosis, } n(\%)(N=5370)\end{array}$ & $4421(82.3)$ \\
\hline $\begin{array}{l}\text { Any opportunistic infections other } \\
\text { than cryptococcosis, } n(\%)\end{array}$ & $910(16.9)$ \\
\hline Anti-HAV IgG, $n(\%)(N=4604)$ & $798(17.3)$ \\
\hline HBsAg, $n(\%)(N=5266)$ & $530(10.1)$ \\
\hline Anti-HCV, $n(\%)(N=5303)$ & $421(7.9)$ \\
\hline $\begin{array}{l}\text { Rapid plasma reagin titer } \geq 1: 4, n(\%) \\
\quad(N=5316)\end{array}$ & $1064(20)$ \\
\hline $\begin{array}{l}\text { Nadir CD4 lymphocyte count, median } \\
\qquad(\mathrm{IQR}), \text { cells } / \mu \mathrm{l}(N=5362)\end{array}$ & $289(132,440)$ \\
\hline$<100, n(\%)$ & $1150(21.4)$ \\
\hline $100-200$ & $708(13.2)$ \\
\hline
\end{tabular}


Table 1 continued

\begin{tabular}{lc}
\hline & $\begin{array}{l}\text { Total } \\
(\boldsymbol{N}=\mathbf{5 3 7 2})\end{array}$ \\
\hline$>200$ & $3504(65.3)$ \\
Plasma HIV RNA load, median & $4.8(4.3,5.3)$ \\
$\quad$ (IQR), $\log _{10}$ copies $/ \mathrm{ml}(N=5329)$ & \\
$>5 \log _{10}$ copies $/ \mathrm{ml}, n(\%)$ & $2158(40.5)$ \\
\hline
\end{tabular}

$I D U$ injection drug user, $I Q R$ interquartile range, $H A V$ hepatitis A virus, $H B s A g$ hepatitis B virus surface antigen, $H C V$ hepatitis $\mathrm{C}$ virus, MSM men who have sex with men

\section{RESULTS}

\section{Characteristics of the Patients}

During the 10-year study period, 5372 newly diagnosed PLWH who sought HIV care at the six participating hospitals for the first time were included, which accounted for $25.2 \%$ of 21,321 newly diagnosed PLWH in Taiwan from 2009 to 2018 [20]. The included PLWH were predominantly male $(97.3 \%)$ and their median age was 29 years (interquartile range [IQR], 25 to 36) (Table 1). The proportion of PLWH who presented with CD $4<100$ and those with $<200$ cells/ $\mu$ l was $21.4 \%$ and $34.7 \%$, respectively, both

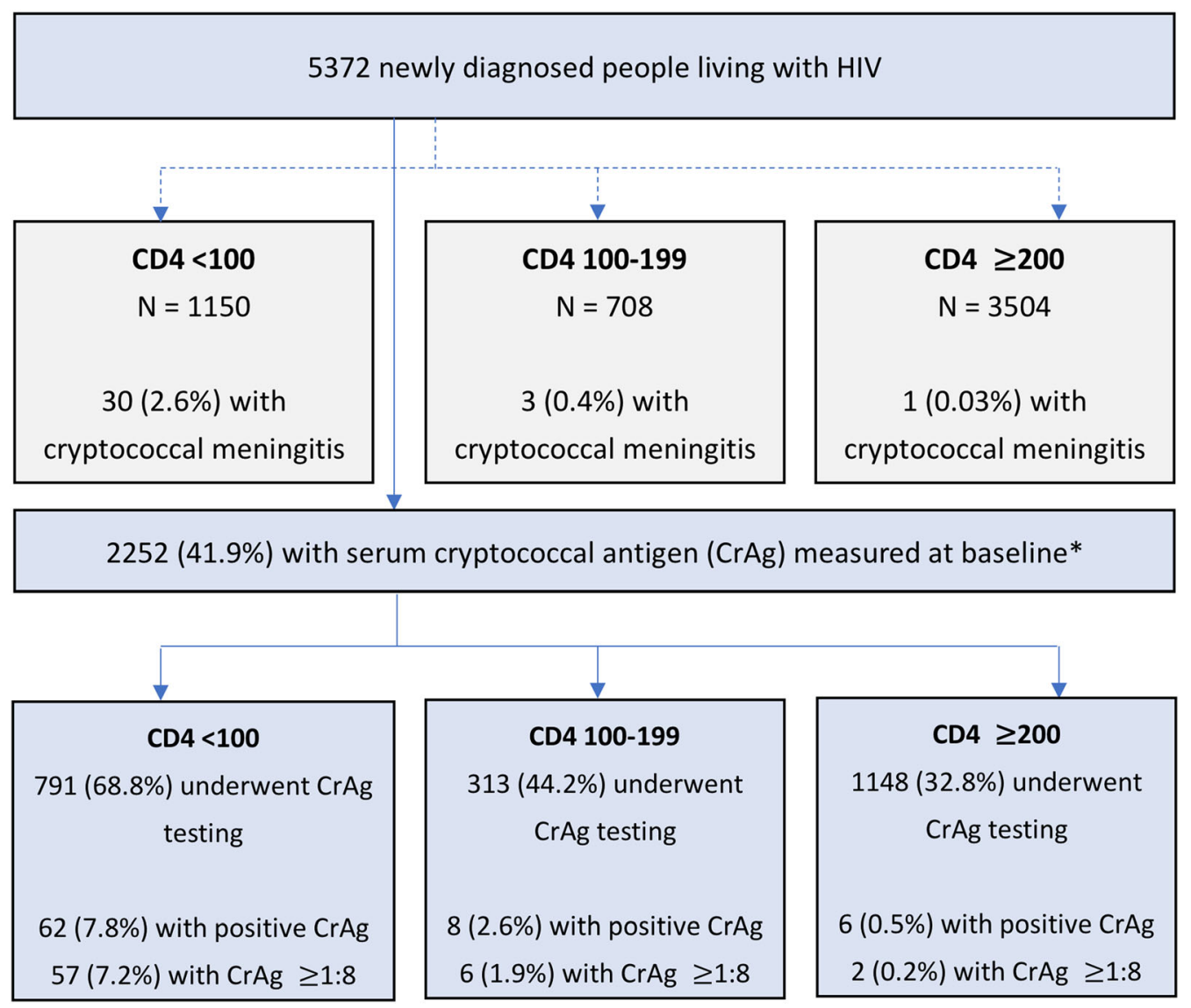

Fig. 1 Prevalence of cryptococcal meningitis and cryptococcal antigenemia. ${ }^{*}$ Among the 5372 newly diagnosed people living with HIV, 2971 (55.3\%) did not have serum cryptococcal antigen measured, $11(0.2 \%)$ had serum $\mathrm{CrAg}$ measured $>6$ months before HIV diagnosis, and 138
(2.6\%) had serum CrAg measured $>6$ months after HIV diagnosis 


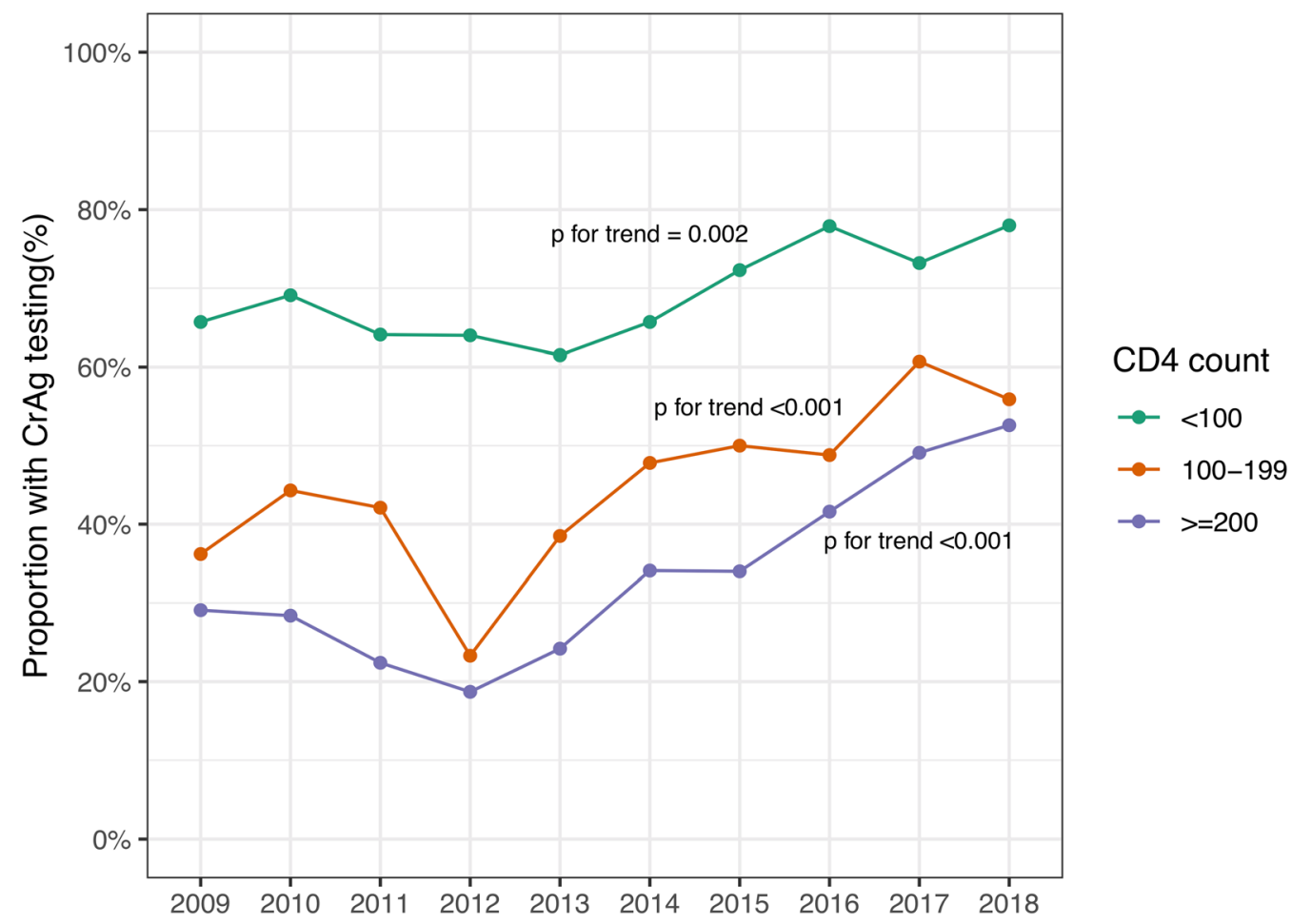

Fig. 2 Annual uptake of serum cryptococcal antigen ( $\mathrm{CrAg}$ ) testing among newly diagnosed people living with HIV according to CD4 lymphocyte count category from 2009 to 2018

of which had decreased over the 10-year study period $(P=0.032$ and 0.039 , respectively) (Fig. S1a, b). More than half $(51.9 \%)$ of the PLWH with baseline CD $<100$ cells $/ \mu \mathrm{l}$ received their HIV diagnosis as outpatients.

\section{Uptake of Cryptococcal Antigen Screening and its Trend}

Overall, $2252(41.9 \%)$ of 5372 PLWH underwent CrAg testing around the time of the HIV diagnosis (Fig. 1). Among PLWH with CD4< 100 cells $/ \mu \mathrm{l}$, the uptake of CrAg testing increased significantly, from $65.7 \%$ in 2009 to $78.0 \%$ in $2018(P=0.002)$ (Fig. 2). The increasing trends were also observed among those with CD4 100-199 and those with CD4 > 200 cells/ $\mu$ l (both with $P<0.001$ ). Among PLWH with CD $4<100$ cells/ $\mu$ l, after exclusion of 289 PLWH who had CrAg testing before their HIV diagnosis, 401 of 861 (46.6\%) underwent CrAg testing within 14 days after confirmed HIV diagnosis, $101 \quad(11.7 \%)$ had $\mathrm{CrAg}$ testing $\geq 14$ days after HIV diagnosis, and 359 (41.7\%) did not have CrAg testing.

Among PLWH who presented with CD4 < 100 cells/ $\mu \mathrm{l}$, those being outpatients at the time of diagnosis were less likely to have CrAg testing (adjusted odds ratio [aOR], 0.313 [95\% confidence interval [CI] 0.225-0.432]), while those who were older (aOR 1.038; 95\% CI, 1.021-1.055), having CD4 lymphocyte counts $<50$ cells $/ \mu \mathrm{l} \quad(\mathrm{aOR}$ 1.643; 95\% CI 1.202-2.245), and with leukocytosis (aOR 2.136; 95\% CI 1.167-4.158) were more likely to have CrAg testing in the multivariate logistic regression model (Table 2 and univariate analysis in Table S1).

\section{Burden of Cryptococcal Disease and its Trend}

Among PLWH who tested for CrAg, the prevalence of cryptococcal antigenemia was $7.8 \%$, $2.6 \%$, and $0.5 \%$ in those with $\mathrm{CD} 4<100$, $100-199, \quad$ and $\geq 200$ cells $/ \mu \mathrm{l}, \quad$ respectively 
Table 2 Factors associated with testing of serum cryptococcal antigen among newly diagnosed people with HIV with nadir CD4 lymphocyte count $<100$ cells $/ \mu$ in multivariate logistic regression model

\begin{tabular}{|c|c|c|}
\hline & $\begin{array}{l}\text { Adjusted odds } \\
\text { ratio }\end{array}$ & $P$-value \\
\hline Age, per 1-year increment & $\begin{array}{l}1.038 \\
\quad(1.021-1.055)\end{array}$ & $<0.001$ \\
\hline \multicolumn{3}{|l|}{ Year of HIV diagnosis } \\
\hline $2012-2013$ vs. $2009-2011$ & $\begin{array}{l}1.048 \\
\quad(0.688-1.597)\end{array}$ & 0.828 \\
\hline $2014-2015$ vs. $2009-2011$ & $\begin{array}{l}1.608 \\
\quad(1.027-2.531)\end{array}$ & 0.039 \\
\hline $2016-2018$ vs. $2009-2011$ & $\begin{array}{l}1.961 \\
\quad(1.286-3.004)\end{array}$ & 0.002 \\
\hline $\begin{array}{l}\text { Being outpatients at the } \\
\text { time of HIV diagnosis }\end{array}$ & $\begin{array}{l}0.313 \\
\quad(0.225-0.432)\end{array}$ & $<0.001$ \\
\hline \multicolumn{3}{|l|}{ White blood cell count } \\
\hline Leukopenia vs. normal & $\begin{array}{l}0.888 \\
\quad(0.646-1.224)\end{array}$ & 0.468 \\
\hline Leukocytosis vs. normal & $\begin{array}{l}2.136 \\
\quad(1.167-4.158)\end{array}$ & 0.018 \\
\hline $\begin{array}{l}\text { CD4 lymphocyte } \\
\text { count }<50 \text { cells } / \mu l\end{array}$ & $\begin{array}{l}1.643 \\
\quad(1.202-2.245)\end{array}$ & 0.002 \\
\hline $\begin{array}{l}\text { Plasma HIV RNA }>5 \log _{10} \\
\text { copies } / \mathrm{ml}\end{array}$ & $\begin{array}{l}1.341 \\
\quad(0.944-1.899)\end{array}$ & 0.099 \\
\hline
\end{tabular}

The bold values indicate a $P$-value $<0.05$

(Fig. 1). The prevalence of cryptococcal antigenemia did not change significantly over the study period, except for the group with CD4 100-199 cells $/ \mu \mathrm{l}$, in which the prevalence decreased over time $(P=0.006)$ (Fig. S2a, b). Among 76 PLWH who tested positive for CrAg, $65(85.5 \%)$ had CrAg titers $\geq 1: 8$, indicating active cryptococcal disease, and 65 (85.5\%) required hospitalization. In a multivariate model, lower CD4 lymphocyte counts, anemia, and being an inpatient at the time of HIV diagnosis were independent factors associated with cryptococcal antigenemia $(P<0.001$, $P=0.012, \quad$ and $\quad P<0.001, \quad$ respectively)
(Table S2). Among all included PLWH, confirmed cryptococcal meningitis occurred in 30 of $1150(2.6 \%)$ PLWH with CD4 < 100 cells/ $\mu \mathrm{l}$, in 3 of $708(0.4 \%)$ with CD4 100-199 cells/ $\mu \mathrm{l}$, and in 1 of $3504(0.03 \%)$ with CD4 $\geq 200$ cells/ $\mu \mathrm{l}$ (Fig. 1). There were no temporal changes in the prevalences of cryptococcal meningitis between 2009 and $2018(P=0.624)$.

\section{Timing of Cryptococcal Antigen Testing and Mortality}

At 6 months of HIV diagnosis, the all-cause mortality rate was $11.8 \%$ among PLWH with positive $\mathrm{CrAg}$ compared to $3.7 \%$ among those with negative CrAg (unadjusted odds ratio 3.516, 95\% CI 1.488-7.417). The overall mortality was highly associated with the CD4 category at the time of HIV diagnosis (Fig. S3). In the Kaplan-Meier plot of PLWH with CD $4<200$ cells/ $\mu \mathrm{l}$, the 6-month mortality was significantly higher among PLWH who were hospitalized at the time of HIV diagnosis compared to those who were not, irrespective of the timing of CrAg testing $(P<0.001$, Figs. $3 \mathrm{a}$ and $\mathrm{S} 4)$. Among those who received HIV diagnosis as outpatients, the mortality in the "late CrAg" group continued to accumulate with time, lasting up to 12 months, and exceeded that in the "early CrAg" and "CrAg before HIV" groups after 2 months of HIV diagnosis $(P=0.027$, Fig. $3 b)$. When the analysis was limited to PLWH presented with $\mathrm{CD} 4<100$ cells $/ \mu \mathrm{l}$, the findings were similar (Fig. S5a, b).

In the Cox proportional hazards model, late $\mathrm{CrAg}$ testing was associated with increased risk of 12 -month mortality compared to early CrAg testing (adjusted hazard ratio [aHR] 2.028, 95\% CI 1.109-3.708) after adjusting for age, risk group of HIV acquisition, year of HIV diagnosis, outpatient/inpatient status and presence of opportunistic infections at the time of HIV diagnosis, baseline CD4 lymphocyte count, and PVL (Table 3). However, of the 12 PLWH with positive CrAg in the "late CrAg" group, only one with concurrent Burkitt's lymphoma died of enterococcal bacteremia at 145 days after HIV diagnosis. 

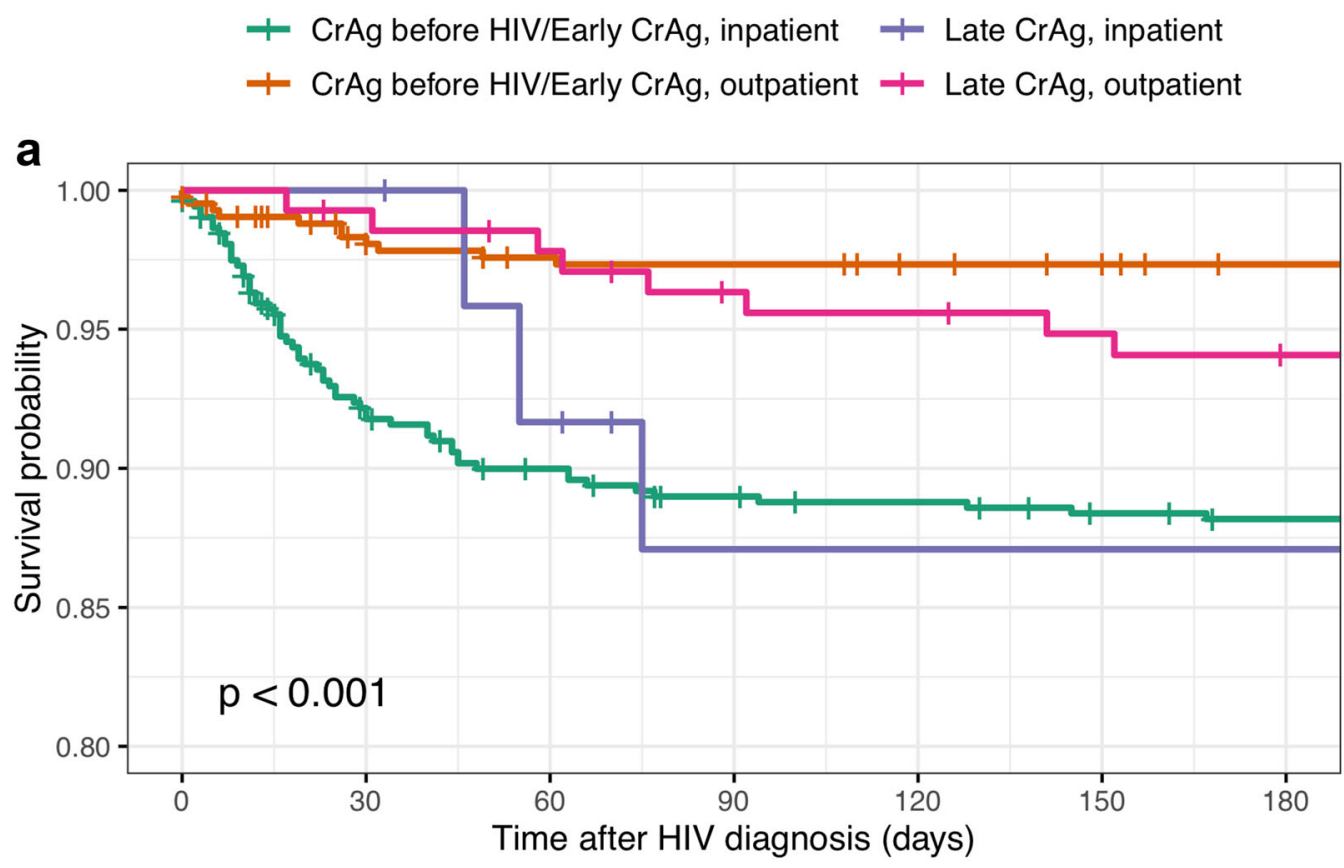

+ CrAg before HIV + Early CrAg + Late CrAg

b

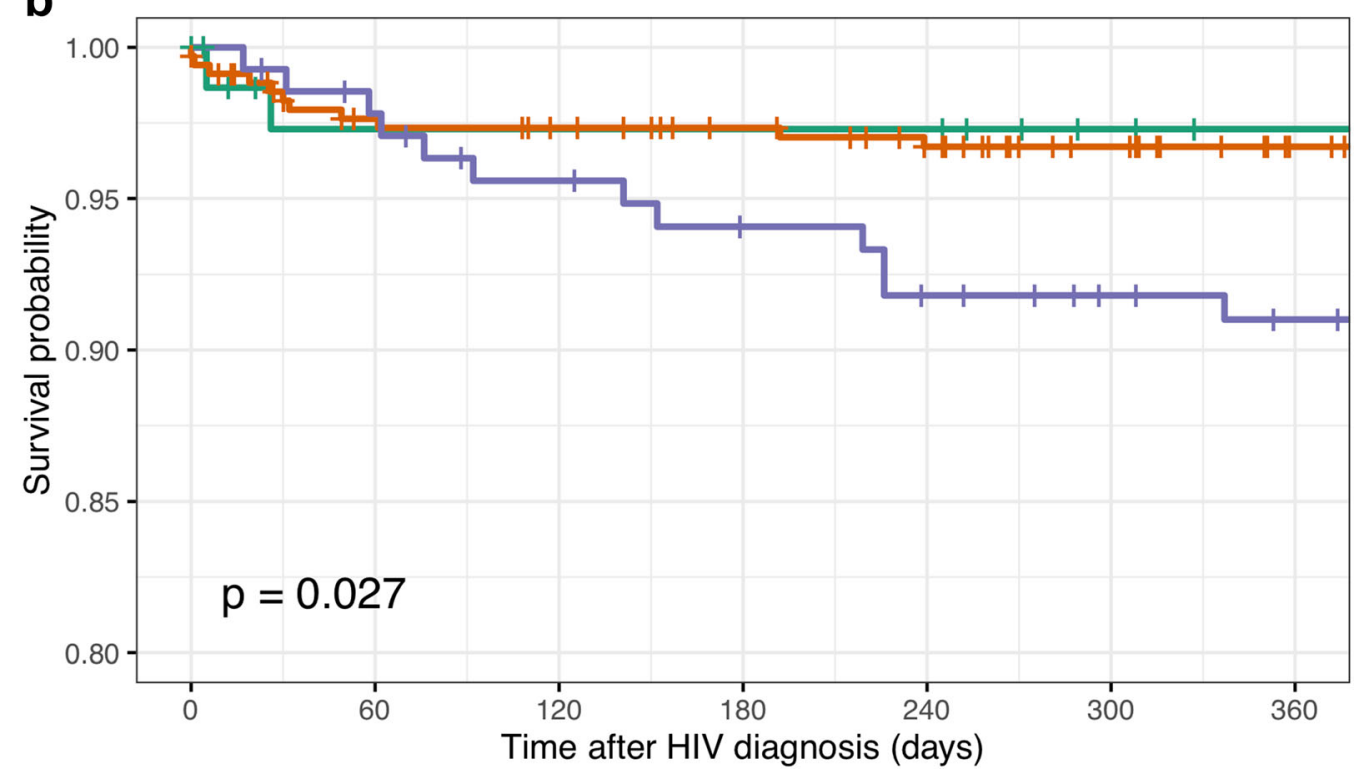

Fig. 3 Kaplan-Meier plot demonstrating. a Six-month allcause mortality by timing of cryptococcal antigen $(\mathrm{CrAg}$ ) testing and outpatient/inpatient status at the time of HIV diagnosis, and $\mathbf{b}$ 12-month all-cause mortality by timing of $\mathrm{CrAg}$ among people living with HIV who tested for $\mathrm{CrAg}$ and presented with initial CD4 lymphocyte count less than 200 cells $/ \mu$ l 
Table 3 Factors associated with 12-month all-cause mortality after HIV diagnosis in the Cox proportional hazards model including 1104 people living with HIV who presented with baseline CD4 lymphocyte count $<200 \mathrm{cells} / \mu \mathrm{l}$ and tested for cryptococcal antigen

\begin{tabular}{llc}
\hline & Adjusted hazard ratio & $\boldsymbol{P}$-value \\
\hline Age, per 1-year increment & $1.030(1.013-1.048)$ & $<\mathbf{0 . 0 0 1}$ \\
Men who have sex with men & $0.505(0.319-0.799)$ & $\mathbf{0 . 0 0 4}$ \\
Year of HIV diagnosis & & \\
2012-2013 vs. 2009-2011 & $0.959(0.554-1.661)$ & 0.882 \\
2014-2015 vs. 2009-2011 & $0.786(0.421-1.471)$ & 0.452 \\
2016-2018 vs. 2009-2011 & $0.749(0.423-1.327)$ & 0.323 \\
Being outpatients at the time of HIV diagnosis & $0.460(0.260-0.816)$ & $\mathbf{0 . 0 0 8}$ \\
Any opportunistic infection & $1.249(0.723-2.157)$ & 0.456 \\
Timing of serum cryptococcal antigen & & \\
Before HIV diagnosis versus within 14 days after HIV diagnosis & $0.992(0.617-1.597)$ & 0.975 \\
14 days and beyond after HIV diagnosis versus within 14 days after HIV diagnosis & $2.028(1.109-3.708)$ & $\mathbf{0 . 0 2 2}$ \\
CD4 lymphocyte count, per $100-$-cell/ $\mu$ increment & $0.331(0.183-0.600)$ & $<\mathbf{0 . 0 0 1}$ \\
Plasma HIV RNA $>$ 5 log 10 copies/ml & $1.224(0.744-2.014)$ & 0.427 \\
\hline
\end{tabular}

The bold values indicate a $P$-value $<0.05$

\section{Timing of Cryptococcal Antigen Testing and Hospitalization}

Among those who presented as outpatients at the time of HIV diagnosis, hospitalizations were also more commonly observed in the "late CrAg" group after 1 month of HIV diagnosis $(P=0.024$, Fig. S6). In a Cox proportional hazards model, late CrAg testing was associated with increased risk for all-cause hospitalization compared to early CrAg testing after adjustments (aHR 1.550, 95\% CI 1.100-2.184).

\section{DISCUSSION}

In this multicenter study, 1 out of 13 newly diagnosed Taiwanese PLWH with a CD4 lymphocyte count $<100$ cells $/ \mu$ l had cryptococcal antigenemia, and this prevalence did not decrease over the 10-year study period. The uptake of CrAg testing among newly diagnosed PLWH was increasing; however, at the end of the study period in $2018,>20 \%$ of newly diagnosed PLWH with CD4 $<100$ cells/ $\mu$ l still missed CrAg testing, while some tested late. Among PLWH with a baseline CD4 count $<200$ cells/ $\mu \mathrm{l}$, late $\mathrm{CrAg}$ testing was associated with increased mortality 2 months after the HIV diagnosis.

Compared with other developed countries that have witnessed a decreasing incidence of HIV-associated cryptococcosis [13], the prevalence of cryptococcal meningitis in Taiwan remained stable despite improved access to HIV care and coverage of ART [21]. This was probably driven by the persistently high number of late presenters throughout the years. In our study, more than a third of newly diagnosed PLWH still presented with CD $4<200$ cells/ $\mu$ l and more than $20 \%$ with CD $4<100$ cells/ $\mu$ l by 2018 (Fi. S1a, b). Compared to the last surveillance data in Taiwan (data up to May 2016) [22], our findings demonstrated that the proportion of late presenters did not decline in the past several years. Ongoing efforts to remove the barriers to early HIV diagnosis and to improve linkage to care are needed to reduce the 
mortality and morbidity related to cryptococcosis and AIDS [1]; meanwhile, in countries with high cryptococcal endemicity like Taiwan [14], other measures to reduce the complications of cryptococcosis in PLWH should be pursued.

The strategy of screening coupled with preemptive antifungal therapy has been shown to prevent cryptococcal meningitis [7] and is costeffective among PLWH with CD $4<100$ cells $/ \mu \mathrm{l}$ [23]. Recently, growing evidence suggests that the risk of cryptococcal disease among PLWH with CD4 100-199 cells/ $\mu$ l was also substantial and should not be overlooked [24-26]. Furthermore, the REMSTART trial demonstrated that mortality could be reduced with $\mathrm{CrAg}$ screening and adherence support among PLWH with $\mathrm{CD} 4<200$ cells $/ \mu \mathrm{l}$ [27]. In the present study, the prevalence of cryptococcal antigenemia among newly diagnosed PLWH with CD4 100-199 was $2.6 \%$, and about $12 \%$ of cryptococcal meningitis developed among PLWH with CD4 $\geq 100$ cells $/ \mu \mathrm{l}$. More studies are needed to investigate the benefit and cost-effectiveness of screening and a pre-emptive treatment strategy in this population with higher CD4 lymphocyte counts.

Our data showed a substantial gap in the uptake of CrAg testing among asymptomatic, newly diagnosed PLWH with advanced immunocompromised state. Among those who presented with $\mathrm{CD} 4<100$ cells $/ \mu \mathrm{l}$ and an opportunity for the screening and a pre-emptive therapy approach (not yet testing for $\mathrm{CrAg}$ at the time of HIV diagnosis), half of them did not test for CrAg or did so within 14 days of their HIV diagnosis, especially those who presented as outpatients. The data from this real-world setting identified a critical gap in terms of HIV care, which has prompted the inclusion of a recommendation regarding $\mathrm{CrAg}$ screening among PLWH with a low CD4 count in the revised Taiwanese national HIV treatment guidelines in 2020 (data not shown). Other innovative strategies, such as reflexive laboratory testing, may address this problem if it can be successfully integrated into the clinical care $[6,26,28]$.

Even with appropriate CrAg screening and pre-emptive fluconazole therapy, the mortality related to HIV-associated cryptococcosis was still higher than that of PLWH without cryptococcosis $[7,29]$. This is not a phenomenon uniquely observed in the resource-limited countries. In Taiwan, where healthcare was generally considered accessible and affordable, our study revealed that the overall mortality of PLWH with cryptococcal antigenemia was more than three times higher compared to that of PLWH who were CrAg negative. Of note, a third of asymptomatic PLWH who screened positive for CrAg had confirmed cryptococcal meningitis $[7,30]$. Post-screening lumbar puncture among those who screen positive for $\mathrm{CrAg}$ is a critical step to identify these patients and to guide optimal treatment. It has been shown that CrAg screening with pre-emptive fungal therapy failed to provide survival benefit when lumber puncture was not provided, especially among patients with high serum CrAg titers [31]. Further research is urgently needed to identify optimal management of this subgroup of patients [32]. In this study, we also found an association of delayed $\mathrm{CrAg}$ testing with increased mortality and hospitalization later after HIV diagnosis. The explanations for the association could be complex. PLWH with delayed CrAg testing could represent those whose ARTs were initiated late or whose adherence to ART was poor, who developed symptoms that triggered diagnostic testing later in the course, and who had the worst outcome, or it could indicate failure of a healthcare system to identify individuals with risks for opportunistic infection, which resulted in increased mortality. As detailed clinical information, such as timing of ART initiation, use of antifungal agents, response to ART, and others, was not included in the analyses in this study, we were unable to better define the reasons behind this observation. Nevertheless, caution should be taken that failure to recognize cryptococcosis early may result in unmasking immune reconstitution inflammatory syndrome after ART initiation, which may link to high mortality [18]. In the era of early and same-day ART, timely screening of $\mathrm{CrAg}$ become even more crucial to reduce cryptococcosis-related detrimental outcomes. 
The study had several limitations. The retrospective study design introduced information and selection biases, which may affect the results of the study. For instance, about $30 \%$ of included PLWH with CD $4<100$ cells/ $\mu$ did not test for CrAg, and the prevalence of cryptococcal antigenemia in this study could be over-estimated. However, even if all of them had negative $\mathrm{CrAg}$, the corrected prevalence would be $5.3 \%$, which would still be comparable to those of other high-burden countries [2]. Outpatient status at the time of HIV diagnosis was used as a surrogate marker for asymptomatic HIV infection. However, to interpret the findings related to this surrogate marker, it would be important to clarify that some of the patients with AIDS or other co-infection could be managed in the outpatient setting.

While the concept of care continuum models has been successfully introduced in HIV, tuberculosis, and HCV care [33], a care cascade for HIV-associated cryptococcosis, including timely screening, performance of lumbar puncture for those who were screened positive, and initiation of antifungals for prevention or treatment depending on the results, could also be implemented to improve clinical care. Like HIV care, the cascade starts with screening. Nowadays, point-of-care lateral flow assay, with better sensitivity and short turn-around time $[34,35]$, has made the screen and pre-emptive strategy more feasible. A newer, semi-quantitative, lateral flow test is currently under development and, if proven in well-designed clinical studies, has a potential to further improve the care cascade [36].

\section{CONCLUSIONS}

The burden of cryptococcosis among newly diagnosed PLWH with advanced immunosuppression remains high in Taiwan. The uptake of CrAg testing is still suboptimal and sometimes delayed. Delayed CrAg testing is associated with increased mortality later in the course of HIV care. Measures should be taken to ensure that people in HIV care also receive timely screening for cryptococcosis and subsequent interventions to reduce the related mortality and morbidity.

\section{ACKNOWLEDGEMENTS}

Funding. The authors received no specific funding for this work. The Rapid Service Fee was funded by the authors.

Authorship. All named authors meet the International Committee of Medical Journal Editors (ICMJE) criteria for authorship for this article, take responsibility for the integrity of the work as a whole, and have given their approval for this version to be published.

Author Contributions. C-CH and Y-TL managed and supervised the study. S-HH, Y-TL, and $\mathrm{C}-\mathrm{CH}$ contributed to the study concept and design. S-HH, C-YL, C-ST, M-ST, C-EL, W-TH, H-AC, W-DL, C-JY, W-Ck, P-LLY-TL, and C-CH were involved in collection and assembly of clinical data. S-HH participated in the data analysis. S-HH, Y-TL and C-CH undertook interpretation of the data and drafted the report.

List of Investigators. The Taiwan HIV Study Group: Hsin-Yun Sun, Yu-Shan Huang, SuiYuan Chang, Pei-Ying Wu, Ling-Ya Chen, JunYu Zhang, Hsi-Yen Chang, Wen-Chun Liu, YiChing Su, and Chien-Ching Hung (National Taiwan University Hospital, Taipei); Ning-Chi Wang and Te-Yu Lin (Tri-Service General Hospital and National Defense Medical Center, Taipei); Kuan-Yin Lin (National Taiwan University Hospital Jin-Shan Branch, New Taipei City); Chia-Jui Yang, Hong-An Chen, and Mao-Song Tsai (Far Eastern Memorial Hospital, New Taipei City); Yi-Chieh Lee (Lotung Poh-Ai Hospital, Lo-Hsu Foundation, I-Lan); Chien-Yu Cheng and Shu-Hsing Cheng (Tao-Yuan General Hospital, Ministry of Health and Welfare, Tao-Yuan); Yi-Chia Huang and Sung-Hsi Huang (National Taiwan University Hospital Hsin-Chu Branch, Hsin-Chu); Yuan-Ti Lee and Jia-Juen Lin (Chung Shan Medical University Hospital, Taichung); Shih-Ping Lin, Chia-Yin Hsieh, and 
Hsiu-Wen Wang (Taichung Veterans General Hospital, Taichung); Mao-Wang Ho (China Medical University Hospital, Taichung); ChungEng Liu and Yu-Lin Lee (Changhua Christian Hospital, Changhua); Guan-Jhou Chen and Chi-Ying Lin (National Taiwan University Hospital Yun-Lin Branch, Yun-Lin); Tung-Che Hung (Ditmanson Medical Foundation Chia-Yi Christian Hospital, Chia-Yi); Hung-Jen Tang and Huei-Chun $\mathrm{Ku}$ (Chi Mei Medical Center, Tainan); Chia-Wen Li, Chin-Shiang Tsai, NanYao Lee, and Wen-Chien Ko (National Cheng Kung University Hospital, Tainan); Po-Liang Lu, Chun-Yuan Lee, and Yen-Hsu Chen (Kaohsiung Medical University Hospital, Kaohsiung); ChenHsiang Lee (Kaohsiung Chang Gung Memorial Hospital, Kaohsiung); Hung-Chin Tsai (Kaohsiung Veterans General Hospital, Kaohsiung); Tun-Chieh Chen (Kaohsiung Municipal TaTung Hospital, Kaohsiung).

Disclosures. Chien-Ching Hung has received research support from Janssen, Merck, Gilead Sciences, and ViiV and speaker honoraria from Abbvie, Bristol-Myers Squibb, Gilead Sciences, and ViiV, and served on advisory boards for Gilead Sciences, Janssen, ViiV, and Abbvie. Hsin-Yun Sun has received research support from Gilead Sciences. Sung-Hsi Huang, ChunYuan Lee, Chin-Shiang Tsai, Mao-Song Tsai, Chun-Eng Liu, Wei-Ting Hsu, Hong-An Chen, Wang-Da Liu, Chia-Jui Yang, Wen-Chien Ko, Po-Liang Lu and Yuan-Ti Lee have nothing to disclose.

Compliance with Ethics Guidelines. The study was approved by the Research Ethics Committees of National Taiwan University Hospital [201003112R] and Far Eastern Memorial Hospital [105040-F] and Institutional Review Boards of Chung Shan Medical University Hospital [CS14034], Changhua Christian Hospital [160408], Kaohsiung Medical University Hospital [KMUH-IRB-20110040], and National Cheng Kung University Hospital [BBR-105-038]. The informed consent was waived. The study was carried out according to the principles expressed in the Declaration of Helsinki and its later amendments.
Data Availability. The datasets generated during and/or analyzed during the current study are available from the corresponding author on reasonable request.

Open Access. This article is licensed under a Creative Commons Attribution-NonCommercial 4.0 International License, which permits any non-commercial use, sharing, adaptation, distribution and reproduction in any medium or format, as long as you give appropriate credit to the original author(s) and the source, provide a link to the Creative Commons licence, and indicate if changes were made. The images or other third party material in this article are included in the article's Creative Commons licence, unless indicated otherwise in a credit line to the material. If material is not included in the article's Creative Commons licence and your intended use is not permitted by statutory regulation or exceeds the permitted use, you will need to obtain permission directly from the copyright holder. To view a copy of this licence, visit http://creativecommons.org/licenses/by$\mathrm{nc} / 4.0 /$.

\section{REFERENCES}

1. Post FA, Szubert AJ, Prendergast AJ, Johnston V, Lyall $\mathrm{H}$, Fitzgerald $\mathrm{F}$, et al. Causes and timing of mortality and morbidity among late presenters starting antiretroviral therapy in the REALITY trial. Clin Infect Dis. 2018;66:S132-9.

2. Rajasingham R, Smith RM, Park BJ, Jarvis JN, Govender NP, Chiller TM, et al. Global burden of disease of HIV-associated cryptococcal meningitis: an updated analysis. Lancet Infect Dis. 2017;17: 873-81.

3. Molloy SF, Kanyama C, Heyderman RS, Loyse A, Kouanfack C, Chanda D, et al. Antifungal combinations for treatment of cryptococcal meningitis in Africa. N Engl J Med. 2018;378:1004-17.

4. Jarvis JN, Leeme TB, Molefi M, Chofle AA, Bidwell G, Tsholo K, et al. Short-course high-dose liposomal amphotericin B for human immunodeficiency virus-associated cryptococcal meningitis: a phase 2 randomized controlled trial. Clin Infect Dis. 2018;68:393-401. 
5. Pasquier E, Kunda J, De Beaudrap P, Loyse A, Temfack E, Molloy SF, et al. Long term mortality and disability in cryptococcal meningitis: a systematic literature review. Clin Infect Dis. 2017;66:1122-32.

6. Faini D, Kalinjuma AV, Katende A, Mbwaji G, Mnzava D, Nyuri A, et al. Laboratory-reflex cryptococcal antigen screening is associated with a survival benefit in Tanzania. J Acquir Immune Defic Syndr. 2019;80:205-13.

7. Temfack E, Bigna JJ, Luma HN, Spijker R, Meintjes $\mathrm{G}$, Jarvis JN, et al. Impact of routine cryptococcal antigen screening and targeted preemptive fluconazole therapy in antiretroviral-naive human immunodeficiency virus-infected adults with CD4 cell counts $<100 / \mu \mathrm{L}$ : a systematic review and metaanalysis. Clin Infect Dis. 2018;68:688-98.

8. Jarvis JN, Harrison TS, Lawn SD, Meintjes G, Wood $\mathrm{R}$, Cleary S. Cost effectiveness of cryptococcal antigen screening as a strategy to prevent HIV-associated cryptococcal meningitis in South Africa. PLOS ONE. 2013;8:e69288-310.

9. Kimaro GD, Guinness L, Shiri T, Kivuyo S, Chanda $\mathrm{D}$, Bottomley $\mathrm{C}$, et al. Cryptococcal meningitis screening and community-based early adherence support in people with advanced human immunodeficiency virus infection starting antiretroviral therapy in Tanzania and Zambia: a cost-effectiveness analysis. Clin Infect Dis. 2019;70:1652-7.

10. Guidelines for the diagnosis, prevention and management of cryptococcal disease in HIV-infectedadults, adolescents and children: supplement to the 2016 consolidated guidelines on theuse of antiretroviral drugs for treating and preventing HIV infection. Geneva: World HealthOrganization; 2018

11. Cogliati M, Prigitano A, Esposto MC, Romanò L, Grancini A, Zani A, et al. Epidemiological trends of cryptococcosis in Italy: molecular typing and susceptibility pattern of Cryptococcus neoformans isolates collected during a 20-year period. Med Mycol. 2018;9:189.

12. Pyrgos V, Seitz AE, Steiner CA, Prevots DR, Williamson PR. Epidemiology of cryptococcal meningitis in the US: 1997-2009. PLoS ONE. 2013;8: e56269.

13. Williamson PR, Jarvis JN, Panackal AA, Fisher MC, Molloy SF, Loyse A, et al. Cryptococcal meningitis: epidemiology, immunology, diagnosis and therapy. Nat Rev Neurol. 2017;13:13-24.

14. Huang Y-S, Denning DW, Shih S-M, Hsiung CA, Wu U-I, Sun HY, et al. Fungal diseases in Taiwan-national insurance data and estimation. J Fungi (Basel). 2019;5:78.
15. Liu W-D, Tsai W-C, Hsu W-T, Shih M-C, Chen M-Y, Sun HY, et al. Impact of initiation of combination antiretroviral therapy according to the WHO recommendations on the survival of HIV-positive patients in Taiwan. J Microbiol Immunol Infect. 2020;53:936-45.

16. Jarvis JN, Lawn SD, Vogt M, Bangani N, Wood R, Harrison TS. Screening for cryptococcal antigenemia in patients accessing an antiretroviral treatment program in South Africa. Clin Infect Dis. 2009;48:856-62.

17. Huang S-H, Chuang Y-C, Lee Y-C, Hung C-C, Sheng $\mathrm{W}-\mathrm{H}, \mathrm{Su} \mathrm{JJ}$, et al. Lumbar puncture for non-HIVinfected non-transplant patients with cryptococcosis: Should it be mandatory for all?. PLoS ONE. 2019; 14:e0221657.

18. Rhein J, Hullsiek KH, Evans EE, Tugume L, Nuwagira E, Ssebambulidde K, et al. Detrimental outcomes of unmasking cryptococcal meningitis with recent ART initiation. Open Forum Infect Dis. 2018;5:2173-7.

19. Nunnari G, Gussio M, Pinzone MR, Martellotta F, Cosentino S, Cacopardo B, et al. Cryptococcal meningitis in an HIV-1-infected person: relapses or IRIS? Case report and review of the literature. Eur Rev Med Pharmacol Sci. 2013;17:1555-9.

20. Taiwan Centers for Disease Control. Statistics of HIV/AIDS [Internet]. [cited 2021 Jan 29]. https:// www.cdc.gov.tw/En/Category/MPage/kt6yloEGURt MQubQ3nQ7pA. Accessed 29 Jan 2021.

21. Taiwan Centers for Disease Control. HIV/AIDS [Internet]. [cited 2021 Jan 29]. https://www.cdc.gov. tw/En/Category/ListContent/bg0g_VU_Ysrgkes_KR UDgQ? uaid=CqNo313w78G1fWhz429xDA. Accessed 29 Jan 2021.

22. Lin K-Y, Cheng C-Y, Li C-W, Yang C-J, Tsai M-S, Liu $\mathrm{C}-\mathrm{E}$, et al. Trends and outcomes of late initiation of combination antiretroviral therapy driven by late presentation among HIV-positive Taiwanese patients in the era of treatment scale-up. PLoS ONE. 2017;12:e0179870.

23. Kaplan JE, Vallabhaneni S, Smith RM, ChideyaChihota S, Chehab J, Park B. Cryptococcal antigen screening and early antifungal treatment to prevent cryptococcal meningitis: a review of the literature. J Acquir Immune Defic Syndr. 2015;68(Suppl 3): S331-9.

24. Tugume L, Rhein J, Hullsiek KH, Mpoza E, Kiggundu R, Ssebambulidde K, et al. HIV-associated cryptococcal meningitis occurring at relatively higher CD4 counts. J Infect Dis. 2018;219:877-83. 
25. Li Y, Huang X, Chen H, Qin Y, Hou J, Li A, et al. The prevalence of cryptococcal antigen (CrAg) and benefits of pre-emptive antifungal treatment among HIV-infected persons with CD4+ T-cell counts $<200$ cells $/ \mu \mathrm{L}$ : evidence based on a metaanalysis. BMC Infect Dis. 2020;20:410-1.

26. Tenforde MW, Milton T, Rulaganyang I, Muthoga C, Tawe L, Chiller T, et al. Outcomes of reflex cryptococcal antigen (CrAg) screening in HIV-positive patients with CD4 counts of $100-200$ cells $/ \mu \mathrm{L}$ in Botswana. Clin Infect Dis. 2020;17:873.

27. Mfinanga S, Chanda D, Kivuyo SL, Guinness L, Bottomley C, Simms V, et al. Cryptococcal meningitis screening and community-based early adherence support in people with advanced HIV infection starting antiretroviral therapy in Tanzania and Zambia: an open-label, randomised controlled trial. Lancet. 2015;385:2173-82.

28. Hurt WJ, Tenforde MW, Molefi M, Mitchell HK, Milton T, Azama MS, et al. Prevalence and sequelae of cryptococcal antigenemia in antiretroviral therapy-experienced populations: an evaluation of reflex cryptococcal antigen screening in Botswana. Clin Infect Dis. 2020 [Online ahead of print].

29. Wake RM, Govender NP, Omar T, Nel C, Mazanderani AH, Karat AS, et al. Cryptococcal-related mortality despite fluconazole preemptive treatment in a cryptococcal antigen screen-and-treat program. Clin Infect Dis. 2019;70:1683-90.

30. Wake RM, Britz E, Sriruttan C, Rukasha I, Omar T, Spencer DC, et al. High cryptococcal antigen titers in blood are predictive of subclinical cryptococcal meningitis among human immunodeficiency virusinfected patients. Clin Infect Dis. 2018;66:686-92.
31. Meya DB, Kiragga AN, Nalintya E, Morawski BM, Rajasingham R, Park BJ, et al. Reflexive laboratorybased cryptococcal antigen screening and preemptive fluconazole therapy for cryptococcal antigenemia in HIV-infected individuals with CD4 $<100$ Cells/ $\mu \mathrm{L}$ : a stepped-wedge, cluster-randomized trial. J Acquir Immune Defic Syndr. 2019;80:182-9.

32. Migone C, Ford N, Garner P, Eshun-Wilson I. Updating guidance for preventing and treating cryptococcal disease: how evidence and decisions interface. Cochrane Database of Syst Rev. 2018;17: P873-5.

33. Perlman DC, Jordan AE, Nash D. Conceptualizing care continua: lessons from HIV, hepatitis $\mathrm{C}$ virus, tuberculosis and implications for the development of improved care and prevention continua. Front Public Health. 2017;4:648-9.

34. Jitmuang A, Panackal AA, Williamson PR, Bennett JE, Dekker JP, Zelazny AM. Performance of the cryptococcal antigen lateral flow assay in non-HIVrelated cryptococcosis. J Clin Microbiol. 2016;54: 460-3.

35. McMullan BJ, Halliday C, Sorrell TC, Judd D, Sleiman S, Marriott D, et al. Clinical utility of the cryptococcal antigen lateral flow assay in a diagnostic mycology laboratory. PLoS ONE. 2012;7: e49541-6.

36. Skipper C, Tadeo K, Martyn E, Nalintya E, Rajasingham R, Meya DB, et al. Evaluation of serum cryptococcal antigen testing using two novel semiquantitative lateral flow assays in persons with cryptococcal antigenemia. J Clin Microbiol. 2020;58:873-6. 